\title{
Multiple Koenen Tumors, a Rare Entity: Combination Treatment with $1 \%$ Topical Sirolimus Electrofulguration and Excision
}

\author{
Vishalakshi Viswanath $^{a, b}$ Jay D. Gupte ${ }^{a}$ Niharika Prabhu $^{c}$ Nilima L. Gour ${ }^{b}$ \\ ${ }^{a}$ Disha Skin and Laser Institute, Thane, India; ${ }^{b}$ Rajiv Gandhi Medical College \& Chhatrapati Shivaji Maharaj Hospital, \\ Thane, India; 'Western India Institute of Neurosciences, Kolhapur, India
}

\section{Established Facts}

- Twenty-nail involvement of Koenen tumors is uncommon and poses a therapeutic challenge.

- Topical mammalian target of rapamycin (mTOR) inhibitors are effective in facial angiofibromas, and oral mTOR inhibitors are useful in visceral tumors of Tuberous Sclerosis Complex.

- Minimal regression has been noted with topical sirolimus in Koenen tumors, and surgical modalities are associated with recurrences.

\section{Novel Insights}

- Topical sirolimus $1 \%$ compounded in white soft paraffin is useful in slowing down the growth and preventing formation of new tumors in multiple Koenen tumors affecting all twenty nails.

- Regression in tumor size before surgical intervention aids complete removal with a good postsurgical outcome.

- Combination of treatment modalities (topical sirolimus $1 \%$ and surgical intervention) reduces recurrences and sustains the remission with good aesthetic and functional outcome.

\section{Keywords}

Koenen tumors · Sirolimus · Electrofulguration · Surgical excision

\section{Abstract \\ Introduction: Koenen tumors are benign, cutaneous mani- festations of tuberous sclerosis. These are disfiguring, pain- ful, and challenging to treat as they frequently recur. We report a case of long-standing, multiple Koenen tumors af- fecting all twenty nails in an elderly female who was suc-}

karger@karger.com www.karger.com/sad cessfully treated with a combination of topical sirolimus $1 \%$, surgical excision, and electrofulguration. Case Report: A 57-year-old lady presented with multiple, asymptomatic periungual, and subungual tumors affecting all twenty nails since 27 years. Cutaneous examination revealed confetti macules, ash-leaf macule, and shagreen patch over trunk. Nail biopsy was compatible with Koenen's tumor. Computerized tomography of brain showed diffuse patchy sclerosis. The tumors were treated with topical sirolimus $1 \%$ ointment for 10 months with excellent regression. Electrofulguration for both great toenails and surgical excision of 
right thumbnail periungual fibroma was done. $1 \%$ sirolimus was advised after the surgical treatment. There were no adverse effects or recurrence of tumors over a 2-year followup. Discussion: Topical sirolimus $1 \%$ was effective in tumor regression and preventing new tumor formation. Larger tumors that interfered in daily chores were treated with excision and electrofulguration. Thus, a combination treatment for this rare presentation of tuberous sclerosis provided optimum results.

c) 2020 S. Karger AG, Basel

\section{Introduction}

Tuberous Sclerosis Complex (TSC) is a genetic disorder characterized by multiple hamartomas of skin, kidney, retina, heart, and central nervous system. Koenen tumors (ungual fibromas) constitute $50 \%$ of cutaneous manifestations and cause cosmetic and functional problems [1]. Therapy is challenging since recurrence rates are high with various treatment modalities. We report a case of multiple Koenen tumors successfully treated with combination of topical sirolimus $1 \%$, simple excision, and electrofulguration.

\section{Case Report}

A 57-year-old housewife presented with multiple growths on finger and toenails since 27 years. She faced pain and difficulty in performing daily tasks as the lesions progressed. On enquiry, she had history of multiple light-colored patches over back. She had no history of epilepsy or any systemic complaints. No other family members were affected. She had received multiple courses of antifungals (oral/topical) in the past. Excision of nail plate was performed 6 years ago followed by recurrence of tumors. Cutaneous examination revealed ash-leaf macule, shagreen patch, and multiple confetti macules on the trunk. Multiple subungual and periungual firm, hyperpigmented to skin-colored growths suggestive of Koenen tumors were present on all the twenty nails of fingers and toes (shown in Fig. 1a, b). Florid growths were present on the hyperkeratotic and dystrophic great toenails. Ophthalmologic examination was normal.

Complete blood count, liver, and renal function tests were normal. Imaging studies including X-rays of both feet and ultrasonography of abdomen were normal. Computerized tomography of the brain showed diffuse patchy sclerotic areas Histopathology showed thickened collagen, scattered fibroblasts, and multiple ectatic blood vessels. Diagnosis of TSC with multiple Koenen tumors was made. She was advised application of $1 \%$ topical sirolimus compounded in white soft paraffin on all the tumors. Initially, oncedaily application for a week was followed by twice daily application without occlusion. Excellent response with $80 \%$ regression of tumors after 10 months was noted (shown in Fig. 2). The hyperkera-
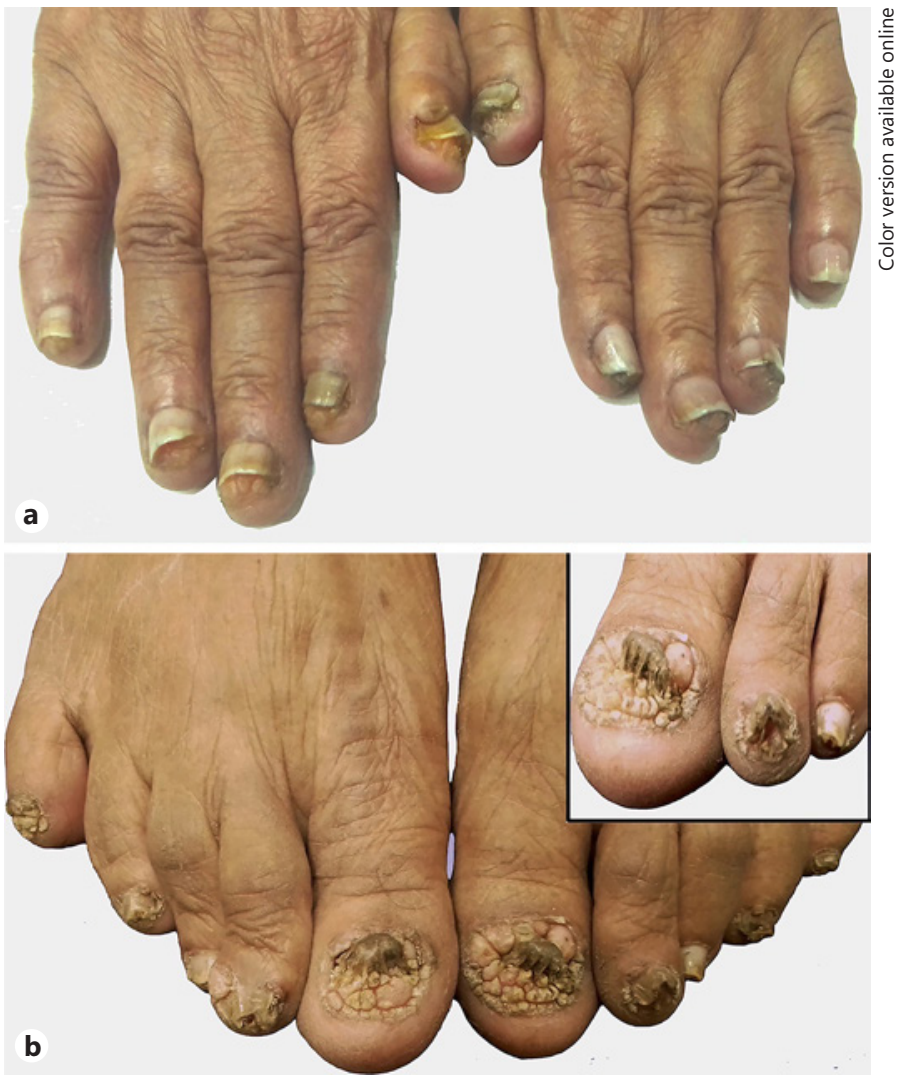

Fig. 1. a Multiple subungual tumors on all fingernails, right thumb showing periungual fibroma. b Multiple florid tumors on all toenails with nail dystrophy. Inset Hyperkeratotic nail plate of the great toe along with florid masses and second toe showing dystrophic nail plates.

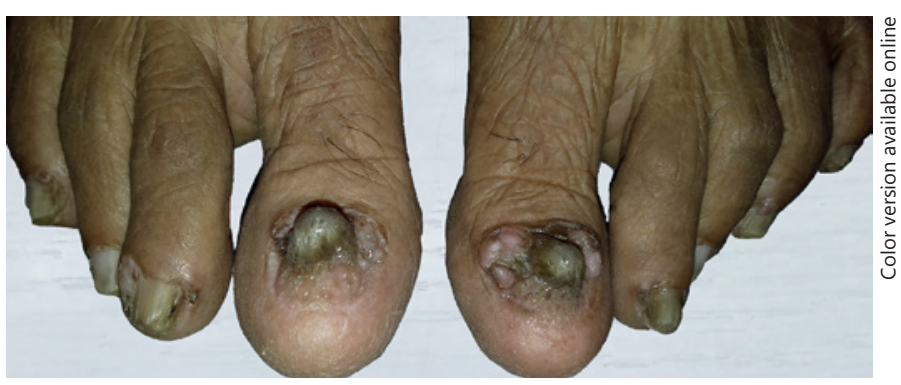

Fig. 2. $80 \%$ regression of tumors after 10 months of $1 \%$ topical sirolimus application with residual dystrophic nails.

totic nail plate of both great toenails was completely avulsed followed by electrofulguration of the residual tumors and the nail beds. Excision of tumor at medial aspect of base of right thumbnail was done as it affected the daily chores of the patient. Good outcome was achieved with the combination treatment of $1 \%$ topical 

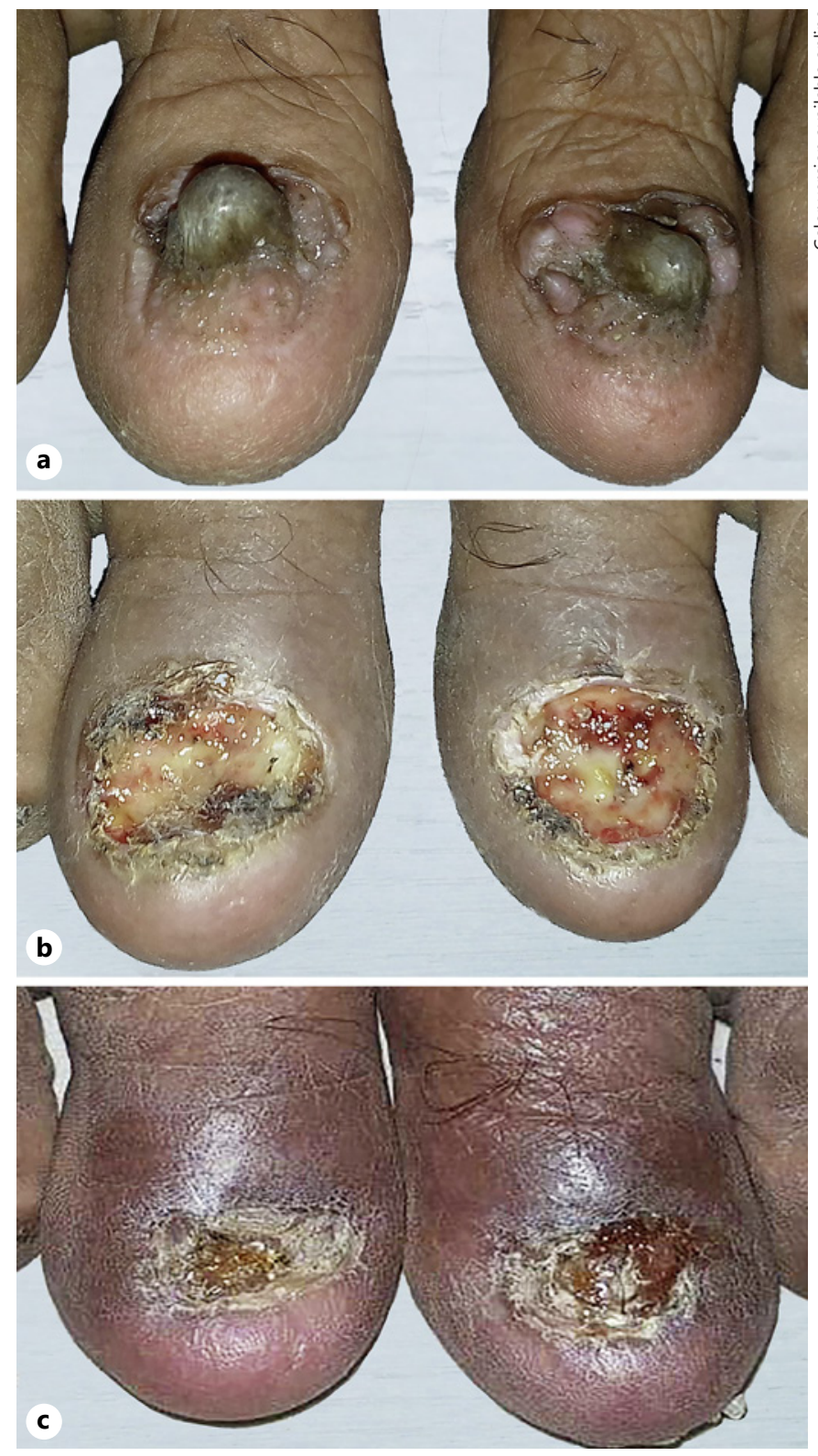

Fig. 3. Hyperkeratotic and dystrophic great toenail after $1 \%$ topical sirolimus (a), 1-month postsurgical nail avulsion along with electrofulguration of tumors and nail beds (b), 2-month postsurgical treatment showing good healing (c).

sirolimus and surgical modalities on the great toenails (shown in Fig. $3 a-c$ ) and right thumb (shown in Fig. $4 a-c)$. The patient was advised to continue $1 \%$ topical sirolimus once-daily postsurgical treatment. She used topical sirolimus for a period of 6 months and later self-discontinued the application. No recurrence of lesions was observed even after 1 year of stoppage of topical sirolimus. Thus, sustained remission with cosmetic and functional improvement was achieved (shown in Fig. 5a, b). No adverse effects were noted.

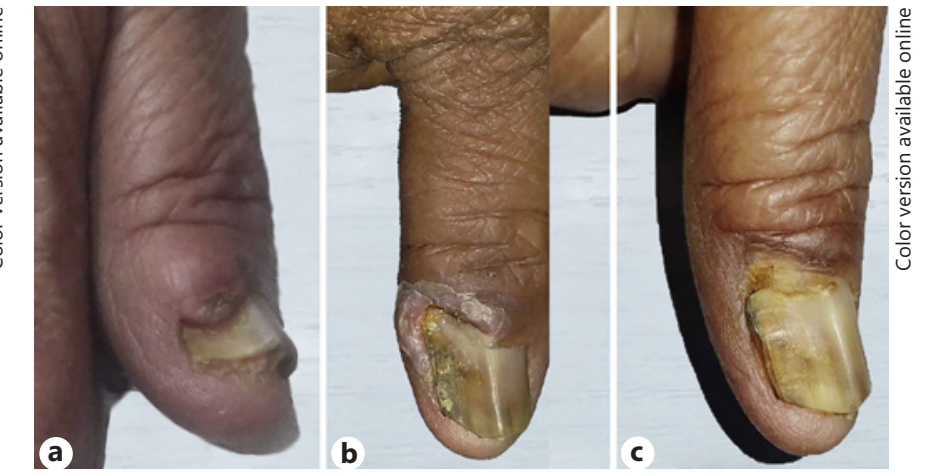

Fig. 4. Residual periungual fibroma on the right thumb after $1 \%$ topical sirolimus (a), 1-month postsurgical excision showing wound healing with intact nail plate (b), 2-month postsurgical treatment with complete healing and good aesthetic outcome (c).

\section{Discussion}

TSC, a genetic autosomal dominant disorder is caused by mutations in tumor suppressor genes. This results in mammalian target of rapamycin (mTOR) disinhibition, and tumor formation. The diagnosis of TSC is interpreted as definite ( 2 major features or 1 major feature with $2 \mathrm{mi}-$ nor features) or possible (1 major feature or 2 minor features) on the basis of diagnostic criteria [2]. Koenen tumor is one of the major criteria. These tumors show fibrosis, stellate fibroblasts, dense collagen, and numerous ectatic blood vessels. Histologically, they may be classified as angiomatous, fibrotic, or mixed subtypes [3]. These tumors are common in females and may manifest at puberty or develop at a later age. Skin-colored or reddish nodules develop on the lateral nail groove, nail plate, or along the proximal nail folds. They are more commonly found on the toes than on the fingers [4]. They may be periungual or subungual in location. Multiple Koenen tumors affecting all digits are uncommon $[1,5-8]$. The size may vary from $1 \mathrm{~mm}$ to $1 \mathrm{~cm}$, and they can sometimes form large masses affecting all the digits [6]. Large florid masses may become confluent and keratotic and are often misdiagnosed and mistreated as fibrokeratomas or periungual warts $[1,5]$. The aggressive lesions may replace the nail plate and lead to local pain, recurrent infections, and difficulty in performing daily activities as seen in this case [1]. Other complications include distal subungual onycholysis, nail dystrophy, and aggressive growths can lead to deformities [1].

Therapeutic modalities include topical sirolimus, dermabrasion, simple excision, ablative $\mathrm{CO}_{2}$ laser, cryo- 


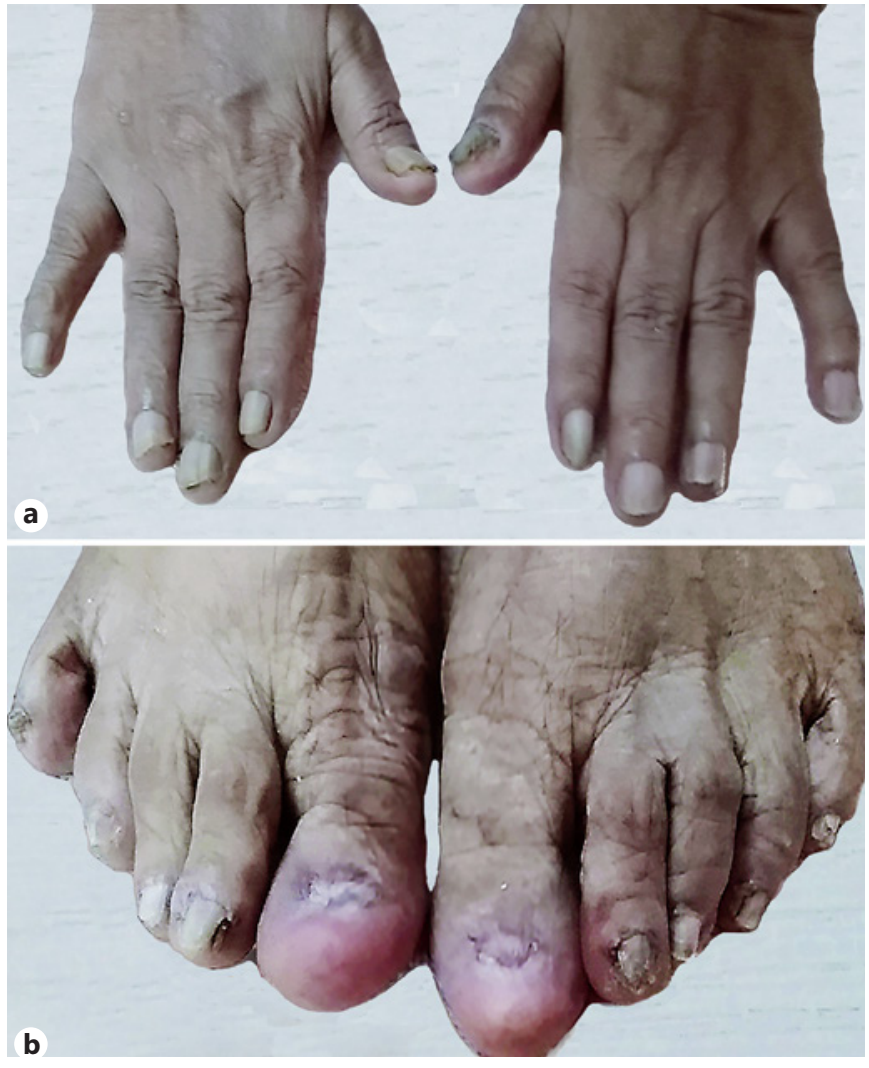

Fig. 5. Two-year follow-up good improvement and aesthetic outcome in all fingernails (a) sustained remission with scarring on great toes $(\mathbf{b})$.

therapy, electrodesiccation, shave, and phenolization [1]. Recurrence rates are high, and there are no standard recommendations among various modalities. Factors considered include age of patient, duration of lesions (recent onset or recurrent), location of tumors (toenails/fingernails), tumor load (low, moderate, or severe), association with cosmetic or functional impairment, and associated comorbidities [7].

The disinhibition of mTOR pathway in TSC promotes tumor growth, and hence, systemic and topical mTOR inhibitors have been useful. Improvement in TSC-related skin lesions including ungual fibromas has been observed when oral sirolimus and everolimus have been used for visceral tumors in TSC [9-11]. Topical sirolimus has been used in varying concentrations and vehicular formulations for facial angiofibromas since 2010. 0.1 and $1 \%$ formulation of sirolimus compounded in white soft paraffin has been found to be a safe, effective, and noninvasive therapy for facial angiofibromas in the Indian skin type [12-14]. In an 18 month prospective study, Malissen et al.
[15] reported good response with $1 \%$ topical sirolimus in facial angiofibromas, fibrous cephalic plaques, and facial hypomelanotic macules. However, minimal regression was seen in $17 \%$ patients ( 1 out of 6 cases) with ungual fibromas [15]. 0.01 and $1 \%$ topical sirolimus has been used by Balak et al. [16] and Muzic et al. [17] for toenail periungual fibromas. Complete clearance of toenails subungual fibromas has been reported with topical sirolimus in a $1 \mathrm{mg} / \mathrm{mL}$ solution form which was applied twice daily under occlusion, for 6 months [17]. In the present case, $1 \%$ topical sirolimus in an ointment base twice daily was effective in reducing the tumor load initially and sustaining the remission postsurgery with once-daily application. Reduction in frequency of application after initial clinical response can ensure patient compliance and costeffectiveness, especially in the Indian context [14]. Recurrence of tumors on discontinuation of topical sirolimus is expected within 2 months and occurs due to the cytostatic rather than cytotoxic effects of sirolimus on the neoplastic cells [14]. Despite self-discontinuation of topical sirolimus, recurrence of tumors after combination treatment was not seen in the present case.

Surgical approach and various ablative techniques are used when there is severe functional and cosmetic impairment. However, recurrences are common with limitations of nail plate conservation and adequate nail growth $[7,18]$. Shave and phenolization, $\mathrm{CO}_{2}$ laser vaporization are treatment options in patients with low tumor burden, severe comorbidities, involvement of fingernails, and when good aesthetic outcome is desired [7]. The advantages are reduced bleeding, short procedural time, preservation of the nail matrix and plate, and good wound healing with cosmetic results [18]. Complications are proximal nail fold necrosis, infection, nail deformity, and recurrences [18]. Excision by surgery, electrofulguration, electrodessication, and ablative radiofrequency is useful in large tumors with distorted nail plates. The technique is simple, cost-effective, easily reproducible and requires less expertise [7]. The disadvantages are higher recurrence rates, difficulty in conservation of proximal fold and nail plate integrity, and pain and prolonged recovery times. In florid tumors, amputation of the nail apparatus and reconstruction with full-thickness skin graft has been described [1]. In the present case, electrofulguration was useful for the hyperkeratotic disfigured nail plates and in areas that involved the nail bed. Surgical excision for the periungual fibromas of fingernails led to better healing with preservation of nail plate and good aesthetic result.

Koenen tumors are often misdiagnosed and mistreated, and a complete history and cutaneous examination 
are important. Early diagnosis of ungual fibromas helps to avoid disfigurement and disability and improves the quality of life. Hence, a possible diagnosis of TSC should be considered when patients present with skin lesions suggestive for TSC, even at an older age. As TSC is a lifelong condition, prolonged use of sirolimus is necessary. However, studies are needed to determine the concentration, frequency of use, and usual time to response and safety profile. This report demonstrates the utility of combination of treatment modalities to reduce the recurrence rate and sustain the remission with good aesthetic results in a patient with multiple Koenen tumors involving all the twenty nails.

\section{Statement of Ethics}

The research presented in the manuscript was conducted ethically in accordance with the World Medical Association Declaration of Helsinki and the appropriate guidelines for human studies. Written informed consent was obtained from the patient for publication of this case report and the accompanying images.

\section{Conflict of Interest Statement}

The authors have no conflicts of interest to declare.

\section{Funding Sources}

There were no funding sources for this work.

\section{Author Contributions}

All the contributors have made substantial contributions to concept and design of this manuscript, acquisition of data, analysis, and its interpretation. All the contributors have worked toward drafting of the article in all aspects and have reviewed the entire manuscript. Dr. Vishalakshi Viswanath has been responsible for the concept, design, data acquisition, and final editing of the intellectual content; Dr. Jay Gupte has performed the surgical interventions and contributed to the figure collages; and Dr. Niharika Prabhu and Dr. Nilima Gour have contributed to the data analysis and manuscript preparation. All the contributors agree to be accountable for all aspects of the article and have given final approval of the version to be published.

\section{References}

1 Oliveira GB, Rossi NCP, Cury DO, Coura MGC, Antonio CR. Exuberant koenen tumors: effective treatment with amputation of the nail apparatus and reconstruction with fullthickness skin grafts. Surg Cosmet Dermatol. 2017;9:187-9. http://dx.doi.org/10.5935/ scd1984-8773.201792834.

2 Northrup H, Krueger DA. International tuberous sclerosis complex consensus group. Tuberous sclerosis complex diagnostic criteria update: recommendations of the 2012 international tuberous sclerosis complex consensus conference. Pediatr Neurol. 2013;49: 243-54. https://dx.doi.org/10.1016\%2Fj.pediatrneurol.2013.08.001.

3 Ma D, Darling T, Moss J, Lee CC. Histologic variants of periungual fibromas in tuberous sclerosis complex. J Am Acad Dermatol. 2011;64(2):442-4.

4 Hake S. Cutaneous manifestations of tuberous sclerosis. Ochsner J. 2010;10(3):200-4.

5 Quist SR, Franke I, Sutter C, Bartram CR, Gollnick HP, Leverkus M. Periungual fibroma (Koenen tumors) as isolated sign of tuberous sclerosis complex with tuberous sclerosis complex 1 germline mutation. J Am Acad Dermatol. 2010;62(1):159-61.

6 Devi B, Dash M, Behera B, Puhan MR. Multiple koenen tumors: an uncommon presentation. Indian J Dermatol. 2011;56(6):773-5.
7 Liebman JJ, Nigro LC, Matthews MS. Koenen tumorsin tuberous sclerosis: a review and clinical considerations for treatment. Ann Plast Surg. 2014;73:721-2. https://doi. org/10.1097/sap.0b013e31828d757d.

8 Aryal E, Bastakoti S, Bhatarria S, Shrestha SB, Pokhrel G. Koenen tumors 20 nail involvement: a rare case report. Ann Clin Case Rep. 2017;2:1411.

9 Nathan N, Wang JA, Li S. Improvement of tuberous sclerosis complex (TSC) skin tumorsduring long-term treatment with oral sirolimus. J Am Acad Dermatol. 2015;73:8028. https://doi.org/10.1016/j.jaad.2015.07.018.

10 Dabora SL, Franz DN, Ashwal S. Multicenter phase 2 trial of sirolimus for tuberous sclerosis: kidney angiomyolipomas and other tumorsregress and VEGF-D levels decrease. PLoS One. 2011;6: e23379. https://doi. org/10.1371/journal.pone.0023379.

11 Franz DN, Budde K, Kingswood JC, Belousova E, Sparagana S, de Vries PJ, et al. Effect of everolimus on skin lesions in patients treated for subependymal giant cell astrocytoma and renal angiomyolipoma: final 4-year results from the randomized EXIST-1 and EXIST-2 studies. J Eur Acad Dermatol Venereol. 2018; 32(10):1796-803
12 Viswanath V, Thakur P, Pund P. Use of topical rapamycin in facial angiofibromas in indian skin type. Indian J Dermatol. 2016;61(1):119.

13 Vasani RJ. Facial angiofibromas of tuberous sclerosis treated with topical sirolimus in an Indian patient. Indian J Dermatol. 2015 MarApr;60(2):165-9.

14 Vasani R. Topical sirolimus in the treatment of facial angiofibromas. Indian J Drugs Dermatol. 2018;4(2):49-51.

15 Malissen N, Vergely L, Simon M, Roubertie A, Malinge MC, Bessis D. Long-term treatment of cutaneous manifestations of tuberous sclerosis complex with topical $1 \%$ sirolimus cream: a prospective study of 25 patients. J Am Acad Dermatol. 2017;77(3):464-e3.

16 Balak DM, Zonnenberg BA, Spitzer-Naaijkens JM, Hulshof MM. A 28-year-old male patient with nail tumors, skin lesions, and epilepsy. Case Rep Dermatol. 2017;9(1):12-9.

17 Muzic JG, Kindle SA, Tollefson MM. Successful treatment of subungual fibromas of tuberous sclerosis with topical rapamycin. JAMA Dermatol. 2014;150(9):1024-5.

18 Mazaira M, Del Pozo Losada J, FernándezJorge B, Fernández-Torres R, Martínez W, Fonseca E. Shave and phenolization of periungual fibromas, Koenen's tumors, in a patient with tuberous sclerosis. Dermatol Surg. 2008;34(1):111-3. 